\title{
Model-Free Control of Shape Memory Alloys Antagonistic Actuators *
}

\author{
Pierre-Antoine Gédouin *, Cédric Join ${ }^{* *, * * *}$, \\ Emmanuel Delaleau*, Jean-Matthieu Bourgeot*, \\ Shabnam Arbab Chirani* , Sylvain Calloch ${ }^{* * * *}$ \\ * Université européenne de Bretagne \\ École nationale d'ingénieurs de Brest \\ Laboratoire brestois de mécanique et des systèmes \\ Technopôle Brest-Iroise, CS 73 862, 29238 Brest, France. \\ e-mail: $\{$ delaleau, bourgeot, gedouin, arbab\}@enib.fr \\ http://www. enib.fr/lrm \\ ** Centre de recherche en automatique de Nancy, UMR CNRS 7039, \\ Université Henry Poincaré-Nancy I, Faculté des sciences et techniques, \\ BP 239, 54506 Vandouvre, France. \\ e-mail: cedric.join@cran.uhp-nancy.fr \\ http://www.cran.uhp-nancy.fr \\ *** Projet ALIEN - INRIA Futurs. \\ e-mail: cedric.join@inria.fr \\ http://www. inria.fr/futurs \\ **** Université européenne de Bretagne \\ École nationale supérieure des ingénieurs \\ des études des techniques d'armement \\ Laboratoire brestois de mécanique et des systèmes \\ 2 rue François Verny, 29806 Brest, France. \\ e-mail: Sylvain.Calloch@ensieta.fr \\ http://www. ensieta.fr/msn
}

\begin{abstract}
This paper deals with a first application of the new framework of model-free control to the promising technology of shape memory alloys actuators. In particular antagonistic shape memory alloys actuator. These devices are known to be difficult to model in a control perspective. Simulations results are exposed and some preliminary experimental results illustrates the paper.
\end{abstract}

Keywords: Nonlinear control design; Robust nonlinear control; Intelligent controllers;

Microactuators; Mechatronic systems

\section{INTRODUCTION}

Shape memory alloys (SMA) offer the possibility to recover a known shape after a thermomechanical cycle. This property, known as the shape memory effect, is due to the transition between the two crystallographic phases they are composed. This variation of shape, controlled by temperature variation, may be used in the development of actuators, see Peirs et al. [2002] and Kohl et al. [2002]. These materials permit to simplify the mechanism, to reduce the size of actuators and to apply force without significant motion, see De Sars [2005]. SMA can easily be heaten by Joule effect, but their control remains a problem and it is principally due to the complexity of their hysteretic behaviour, see Patoor et al. [1994]. A Second difficulty is that the characteristics of the material are time-varying, especially during cyclic loadings. Phases

\footnotetext{
* This work was supported in part by the Agence nationale de la recherche (France) — Project "MAFESMA", in part by Brest métropôle océane, in part by Conseil génénéral du Finistère. P.-A. Gédouin is partially supported by Brest métropôle océane.
}

kinetic transformations and 3-dimensional models are proposed in Leclerc and Lexcellent [1996], Sittner et al. [2000], Arbab Chirani et al. [2003] and Bouvet et al. [2004]. These complex models can render very subtle properties of SMA, but often need to compute a finite element code, what is not suitable for control. On the opposite side, Robotics research have been done on SMA actuator by using simpler model and classical control method. Classical PID loop are used in Calin et al. [1997], Shameli et al. [2005] and Da Silva [2007]. A feedforward path is added in Majima et al. [2001]; the feedforward command is obtain by using a Preisasch model for modelling the hysteretic effect. In Dutta et al. [2005] feedforward scheme is also used but the hysteretic behaviour is described by a Duhem differential hysteresis model. Passivity property of the system is used in Madill and Wang [1998] to prove stability of a proposed proportional law. Nonlinear control techniques based on the Lie algebra are also used in Benzaoui et al. [1999]. Even if their model are quite good enough, using a dynamic model for computing the control law, need firstly to identify the model parameter. As we have already mention the 
model parameter of SMA vary during cycling, then a classical model based control is ineffective or particularly complex. We report our experience, where industrial partners still explain that in order to realise the process control, the part of process modelling, represents $90 \%$ of project global time. Indeed, to define the physical model structure, to identify unknowns parameters, to collect experimental data, to valid the model are never simple. However, how is it possible to control a complex process without any model?

In this paper, a solution to this difficult problem is proposed. The proposition is based on some new results in this framework (Fliess et al. [2006b,a], Join et al. [2006]). The approach uses a derivative estimation (see, Mboup et al. [2007], Fliess et al. [2007]) which provides a good results even if signal are corrupted by noise. Thus a non-physical model valid a very short part of time is estimated and permits classical control design.

Some simulations highlight the advantages of the proposed method. To this end, several perturbation types are simulated. This is particularly clear in case of SMA, where model complexity is detailed.

The paper is organised as follows: The next Section is a short review and introduction to the new "Model-free control" and explains the design of a control law with this new framework; Sec. 3 develops the model-free control of a shape memory alloys actuator and gives simulation results. Sec. 4 concludes the paper and raises some perspectives.

\section{MODEL FREE CONTROL}

Model free control is a very recent approach to nonlinear control that has been introduced in [Fliess et al., 2006a] and [Fliess et al., 2006b]. A first industrial and convincing application is reported in [Join et al., 2006].

\subsection{Derivatives of noisy signals}

Firstly, we recall basic of derivative estimation. Interested reader might refer to [Mboup et al., 2007] for a complete presentation.

Consider a real-valued time function $x$ which is assumed to be analytic on some interval $t_{1} \leqslant t \leqslant t_{2}$. For the sake of simplicity, assume $x$ is analytic around $t=0$ and introduce its truncated Taylor expansion:

$$
x(t)=\sum_{\nu=0}^{N} x^{(\nu)}(0) \frac{t^{\nu}}{\nu !}+o\left(t^{N}\right)
$$

Approximate $x(t)$ in the interval $[0, \varepsilon], \varepsilon>0$, by a polynomial $x_{N}(t)=\sum_{\nu=0}^{N} x^{(\nu)}(0) \frac{t^{\nu}}{\nu !}$ of degree $N$. The usual rules of symbolic calculus in Schwartz's distributions theory yields

$$
x_{N}^{(N+1)}(t)=x(0) \delta^{(N)}+\dot{x}(0) \delta^{(N-1)}+\cdots+x^{(N)}(0) \delta
$$

where $\delta$ is the Dirac measure at 0 . From $t \delta=0, t \delta^{(\alpha)}=$ $-\alpha \delta^{(\alpha-1)}, \alpha \geqslant 1$, we obtain the following triangular system of linear equations for determining estimated values $\left[x^{(\nu)}(0)\right]_{e}$ of the derivatives ${ }^{1} x^{(\nu)}(0)$ :

\footnotetext{
1 Those quantities are linearly identifiable Fliess and Sira-Ramirez [2003].
}

$$
\begin{aligned}
& t^{\alpha} x^{(N+1)}(t)=t^{\alpha}\left([x(0)]_{e} \delta^{(N)}\right. \\
& \left.+[\dot{x}(0)]_{e} \delta^{(N-1)}+\cdots+\left[x^{(N)}(0)\right]_{e} \delta\right) \\
& \alpha=0, \ldots, N
\end{aligned}
$$

The time derivatives of $x$ and the Dirac measures and its derivatives are removed by integrating with respect to time both sides of equation (1) at least $N$ times:

$$
\begin{aligned}
\int^{(\nu)} \tau_{1}^{\alpha} x^{(N+1)}\left(\tau_{1}\right)= & \int^{(\nu)} \tau_{1}^{\alpha}\left([x(0)]_{e} \delta^{(N)}\right. \\
& \left.+[\dot{x}(0)]_{e} \delta^{(N-1)}+\cdots+\left[x^{(N)}(0)\right]_{e} \delta\right) \\
& \nu \geqslant N, \alpha=0, \ldots, N
\end{aligned}
$$

where $\int^{(\nu)}=\int_{0}^{t} \int_{0}^{\tau_{\nu-1}} \ldots \int_{0}^{\tau_{1}}$ is an iterated integral. A quite accurate value of the estimates may be obtained with a small time window $[0, t]$.

\subsection{Nonlinear systems}

Consider the generalised state representation of a MIMO (multi-inputs multi-outputs) nonlinear system

$$
\begin{aligned}
& A_{\iota}\left(\dot{x}_{\iota}, \mathbf{x}, \mathbf{u}, \ldots, \mathbf{u}^{(\alpha)}\right)=0 \\
& B_{\kappa}\left(y_{\kappa}, \mathbf{x}, \mathbf{u}, \ldots, \mathbf{u}^{(\beta)}\right)=0
\end{aligned}
$$

where $A_{\iota}, \iota=1, \ldots, n, B_{\kappa}, \kappa=1, \ldots, p$, are nonlinear functions, $\mathbf{x}=\left(x_{1}, \ldots, x_{n}\right)$ the state vector, $\mathbf{u}=$ $\left(u_{1}, \ldots, u_{m}\right)$ the input control vector, $\mathbf{y}=\left(y_{1}, \ldots, y_{p}\right)$ the output (or measured signals) vector.

It exists a set of nonlinear functions $\Phi_{j}, j=1, \ldots, p$, generally called input-output representation

$$
\Phi_{j}\left(\mathbf{y}, \ldots, \mathbf{y}^{\left(\bar{N}_{j}\right)}, \mathbf{u}, \ldots, \mathbf{u}^{\left(\bar{M}_{j}\right)}\right)=0
$$

with appropriate orders $\bar{N}_{j}, \bar{M}_{j}$.

\subsection{Local model}

We now introduce a local non physical model on which the model-free control will be developed in the next Section.

$$
\begin{aligned}
& y_{1}^{\left(n_{1}\right)}=F_{1}+\alpha_{1,1} u_{1}+\cdots+\alpha_{1, m} u_{m}+\beta_{1} \\
& \vdots \\
& y_{p}^{\left(n_{p}\right)}=F_{p}+\alpha_{p, 1} u_{1}+\cdots+\alpha_{p, m} u_{m}+\beta_{p}
\end{aligned}
$$

where,

- $\bar{N}_{j} \geqslant n_{j} \geqslant 1, j=1, \ldots, p$;

- $F_{j}, \alpha_{j, i}, \beta_{j} \in \mathbb{R}, i=1, \ldots, m, j=1, \ldots, p$, are nonphysical constant parameters free of choice;

- if $p>m, m$ outputs are selected to insure invertibility property, i.e.

$$
\operatorname{det} \gamma=\left(\begin{array}{ccc}
\alpha_{1,1} & \cdots & \alpha_{1, m} \\
\vdots & \ddots & \vdots \\
\alpha_{m, 1} & \cdots & \alpha_{m, m}
\end{array}\right)
$$

To realise previous choices, some practical remarks are now given. They can be viewed like facilitating the implementation but not as restriction cases.

Remark 2.1. The smaller the integers $n_{j}$ in (3) are, the better it is for the usefulness of the local model.

Remark 2.2. It is better to have as many zeros as possible on each row of the matrix $\gamma$. 
Remark 2.3. The lower the orders of derivation of $u$ are in $\Phi_{j}$ in (2), the better it is for the local model.

Here, we do not search to estimate $F_{j}$ according physical knowledge but rather than totally unknown.

Thus, using derivative estimation $\left[y_{j}^{\left(n_{j}\right)}\right]_{e}$ of $y_{j}^{\left(n_{j}\right)}$ we obtain the explicit estimation of $F_{j}$

$$
\left[F_{j}(t)\right]_{e}=\left[y_{j}^{\left(n_{j}\right)}\right]_{e}-\sum_{i=1}^{m} \alpha_{j, i} u_{i}-\beta_{j}
$$

where all signals are known as constants.

To avoid algebraic loop and since numerical controller implementation are sampled

$$
\left[F_{j}(\kappa)\right]_{e}=\left[y_{j}^{\left(n_{j}\right)}(\kappa)\right]_{e}-\sum_{i=1}^{m} \alpha_{j, i} u_{i}(\kappa-1)-\beta_{j}
$$

where $[\bullet(\kappa)]_{e}$ stands for an estimation at time $\kappa$.

To conclude, let us insist on the fact that we use a local model estimated at each sampled time and none model structure is particularly considered. Indeed, function of time $F_{j}$ is unspecified, i.e., without particularity.

\subsection{Model-free control design}

According to previous estimation, control law is divided into two parts,

$$
\mathbf{u}=\mathbf{u}_{\mathbf{p}}+\mathbf{u}_{\text {flat }}
$$

where $\mathbf{u}_{\mathbf{p}}$ rejects the estimated model part which can be viewed as a model perturbation and $\mathbf{u}_{\text {flat }}$ is classical flatness-based control (see, e.g., Fliess et al. [1995], Hagenmeyer and Delaleau [2003b,a]). At the last part is also associated a GPI (see Fliess et al. [2002]).

Model perturbation can be rejected thanks to

$$
\mathbf{u}_{\mathbf{p}}(\kappa)=-\left(\begin{array}{ccc}
\alpha_{1,1} & \cdots & \alpha_{1, m} \\
\vdots & \ddots & \vdots \\
\alpha_{m, 1} & \cdots & \alpha_{m, m}
\end{array}\right)^{-1}\left(\begin{array}{c}
{\left[F_{1}(\kappa)\right]_{e}+\beta_{1}} \\
\vdots \\
{\left[F_{m}(\kappa)\right]_{e}+\beta_{m}}
\end{array}\right)
$$

Using this part of control (3) is rewritten

$$
\left(\begin{array}{c}
y_{1}^{\left(n_{1}\right)} \\
\vdots \\
y_{p}^{\left(n_{p}\right)}
\end{array}\right)=\left(\begin{array}{ccc}
\alpha_{1,1} & \cdots & \alpha_{1, m} \\
\vdots & \ddots & \vdots \\
\alpha_{m, 1} & \cdots & \alpha_{m, m}
\end{array}\right) \mathbf{u}_{\text {flat }}
$$

One possibility is then to set

$$
\begin{aligned}
\mathbf{u}_{\text {flat }}= & \left(\begin{array}{ccc}
\alpha_{1,1} & \cdots & \alpha_{1, m} \\
\vdots & \ddots & \vdots \\
\alpha_{m, 1} & \cdots & \alpha_{m, m}
\end{array}\right)^{-1}\left(\begin{array}{c}
y_{1}^{\star\left(n_{1}\right)} \\
\vdots \\
y_{p}^{\star\left(n_{p}\right)}
\end{array}\right) \\
& +\left(\begin{array}{cccc}
\delta_{1} & 0 & \cdots & 0 \\
0 & \ddots & \ddots & \vdots \\
\vdots & \ddots & \ddots & 0 \\
0 & \cdots & 0 & \delta_{m}
\end{array}\right)\left(\begin{array}{c}
y_{1}^{\left(n_{1}\right)}-y_{1}^{\star\left(n_{1}\right)} \\
\vdots \\
y_{p}^{\left(n_{p}\right)}-y_{p}^{\star\left(n_{p}\right)}
\end{array}\right)
\end{aligned}
$$

where $y_{i}^{\star}$ is the reference trajectory that must be tracked by $y_{i}$. The diagonal matrix $\delta=\operatorname{diag}\left(\delta_{1}, \cdots, \delta_{m}\right)$ is composed by rational function of polynomial in undetermined $s$ such that all transfer functions $s^{n_{i}}-\delta(s)$ are stable. It ensures to reject estimation error of $F_{j}$ and that tracking error decreases to zero.

\section{CONTROL OF THE SMA ANTAGONISTIC ACTUATOR}

Note that in this paper, the system model is important to ensure realistic simulations. Moreover due to his model complexity, SMA Antagonistic Actuator is a relevant example.

However, in a practical way, interest of the previous control strategy is to forgive the following section.

\subsection{Model of SMA Antagonistic Actuator}

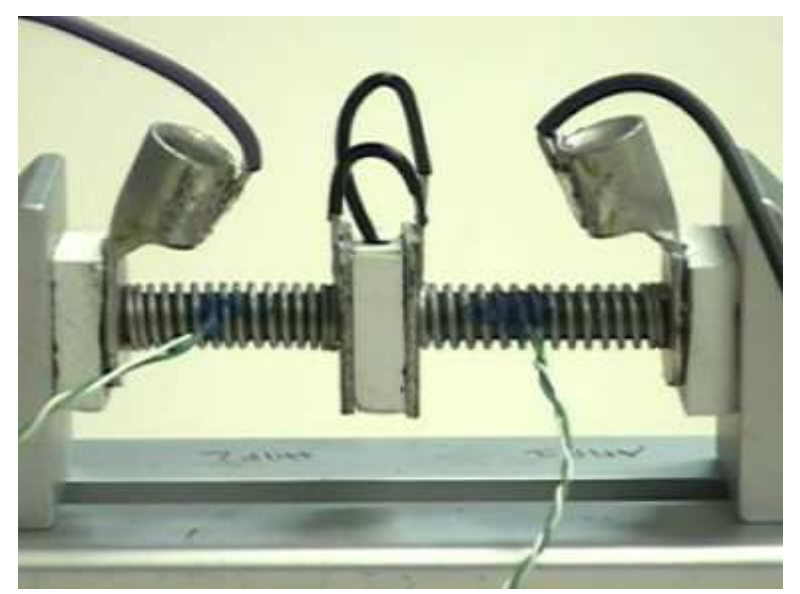

Fig. 1. Picture of the SMA Antagonistic Actuator

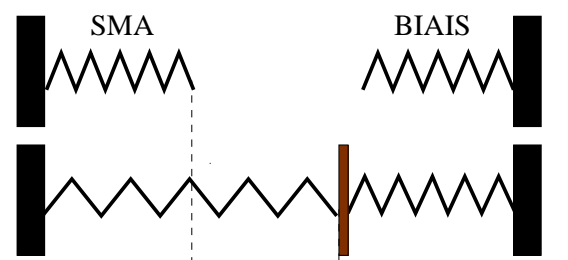

(a)

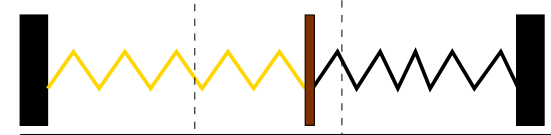

(b)

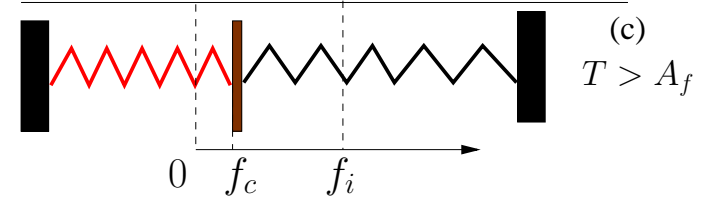

Fig. 2. Notations for the SMA spring actuator

We consider an actuator which consists of a SMA spring and a bias spring as in Fig. 1. The mechanism of this kind of actuator is decomposed into three steps. Firstly, when the temperature is constant and lower than the austenite start temperature $A_{s}$, the SMA spring is purely martensitic and the tensile force provided by the bias spring deforms the SMA spring to an initial deflection denoted by $f_{i}$, on Fig. 2-(a). Secondly the spring is heated by Joule effect, see Fig. 2-(b). To describe the evolution of the temperature as a function of the input electric power, we use equation (7) which is the simplified heat transfer equation proposed in Madill and Wang [1998].

$$
\frac{d T}{d t}=-\frac{T-T_{e}}{\tau}+\frac{P}{m C}
$$


Where $P$ is the control input and corresponds to the electric power crossing the SMA spring, $\tau$ is the characteristic time of the SMA spring, $m$ the mass of the spring, $T_{e}$ the ambient temperature and $C$ the specific heat of the SMA spring. When the temperature of the SMA spring goes past $A_{s}$, the martensite begins to change into austenite, so the spring tries to achieve his high temperature shape, during this phase we proposed to describe the deflection of the SMA spring as a function of the temperature as Fig. 3. This leads to a nonlinear control model.

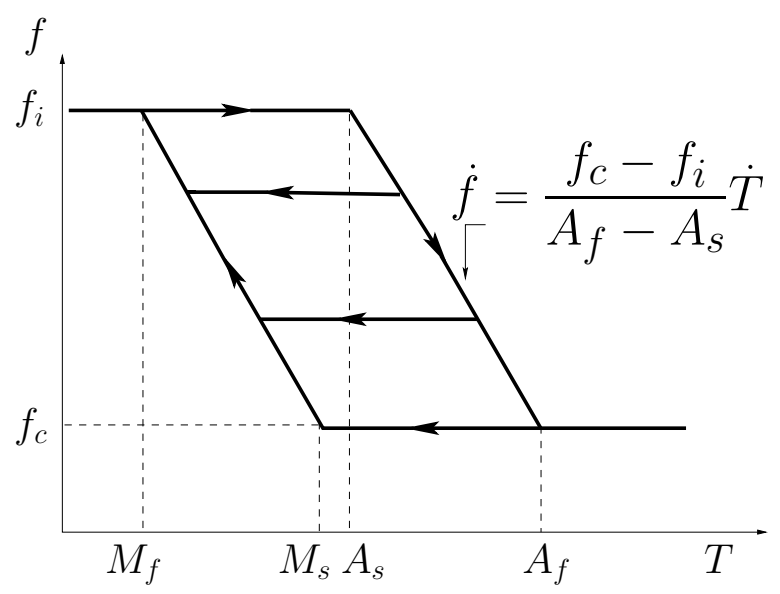

Fig. 3. Deflection-temperature hysteresis

When the temperature goes past the austenite finish temperature $A_{f}$, the persisted deflection denoted by $f_{c}$, on Fig. 2-(c), corresponds to austenite elasticity. Finally, when the spring is cooled lower than the martensite start temperature $M_{s}$ (by free convection for example), the austenite changes into martensite and the effort provided by the bias spring permits the SMA spring to recover its low temperature shape. When the temperature reach the martensite finish temperature $M_{f}$, the spring is purely martensitic and the deflection reach the initial deflection $f_{i}$.

\subsection{Control development}

For this example the very simple first-order local model

$$
\dot{y}=F+\alpha u
$$

is considered. Here, we choose $\alpha=0.5$ and at $u$ corresponds the electric power crossing the SMA spring $(P)$.

$$
u=\frac{1}{\alpha}\left(-[F(\kappa)]_{e}+\dot{y}^{\star}+K_{p}\left(y-y^{\star}\right)+K_{I} \int\left(y-y^{\star}\right)\right)
$$

where $K_{p}$ and $K_{I}$ ensures that $s-K_{P}-\frac{K_{I}}{s}$ is stable transfer function.

\subsection{Numerical simulations}

Simulation parameters are given table 1 .

Several simulation scenarios are considered. For each, figures 4 to 9 , we present estimation needed to compute the control at each time :

- output derivative, computed in presence of an uniform noise in [-0.05, 0.05],

- estimation of local model according $F$,
- temperature evolution,

- control input $u$,

- output tracking.

\section{Scenario 1 (figure 4):}

We present classical tracking. Note that our results can be favourably compared with results which would be obtained using exact knowing of model.

\section{Scenario 2 (figure 5):}

To test approach robustness, an abrupt change of $T$ is simulated by the addition of bias of magnitude equals to $-10^{\circ} \mathrm{C}$ (see figure $5-(\mathrm{c})$ ). Control rejects easily this perturbation.

\section{Scenario 3 (figure 6):}

Another type of perturbation is simulated. At time $t=$ $50 s, \tau$ change from 60 to 120 . One more time, this important parametric change does not affect tracking trajectory. Model based control can be significantly corrupted.

\section{Scenario 4 and 5 (figures 7 and 8):}

Firstly, position decreasing satisfactorily thanks to a rather fast natural cooling. Secondly, the problem is getting more difficult to solve if the ambient temperature would be a little bit larger. This would slow the cooling effect. In this context, the eventual negative saturation of the control could be handled with a classical anti-windup devices added in the controller (see, e.g., Hippe [2006]).

\section{Scenario 6 (figures 9):}

To conclude, we change the trajectory profile and we want now a very fast respond. Control gives still waited results.

\section{CONCLUSION}

The contribution of the paper is twofold: Firstly, it presents a convincing application of the new free-model control in the area of SMA actuators control, a field in which control-model is especially difficult to obtain.

Secondly, in order to be able to present simulation results, the paper exposes a simplified model of the SMA Antagonistic Actuator. This model has been validated experimentally and works pretty well in situation where the behaviour is stabilised, i.e. is not varying along various thermomechanical cycles. However, in practise, the behaviour of SMA shows important hysteresis.

\begin{tabular}{|c|c|c|c|}
\hline Parameter*** & Value & Parameter** & Value \\
\hline$C m\left(\mathrm{~W} /{ }^{\circ} \mathrm{Cs}\right)$ & $2.7 \times 10^{-4}$ & $A_{s}\left({ }^{\circ} \mathrm{C}\right)$ & 42 \\
\hline$\tau(\mathrm{s})$ & 60 & $A_{f}\left({ }^{\circ} \mathrm{C}\right)$ & 75 \\
\hline Parameter* & Value & Parameter** & Value \\
\hline$f_{i}(\mathrm{~mm})$ & 7 & $M_{s}\left({ }^{\circ} \mathrm{C}\right)$ & 53 \\
\hline$f_{c}(\mathrm{~mm})$ & 3.5 & $M_{f}\left({ }^{\circ} \mathrm{C}\right)$ & 44 \\
\hline
\end{tabular}

Table 1. Simulation parameters obtain from spring model $(*)$ DSC measurements $(* *)$ and heating tests $(* * *)$ 


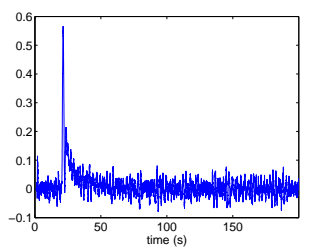

(a) Derivative estimation (b) Model part estimation $[\dot{y}]_{e}$

$[F]_{e}$

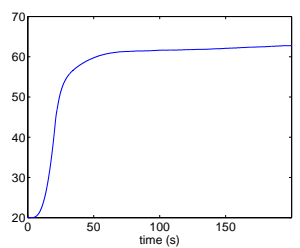

(c) Temperature $T$

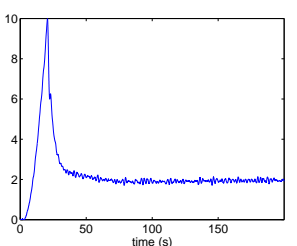

(d) Control input

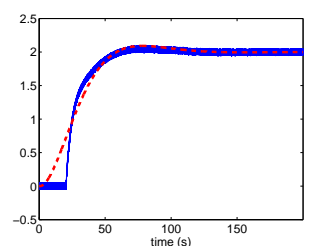

(e) Outputs (-), reference (-)

Fig. 4. Without perturbation

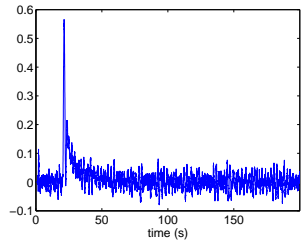

(a) Derivative estimation (b) Model part estimation $[\dot{y}]_{e}$ $[F]_{e}$

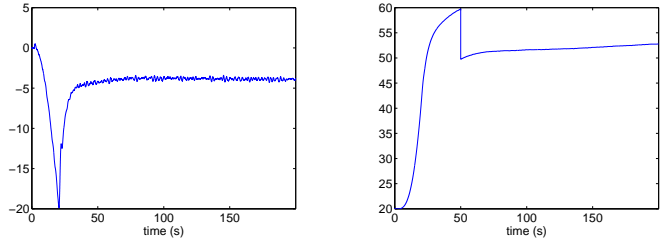

(c) Temperature $T$

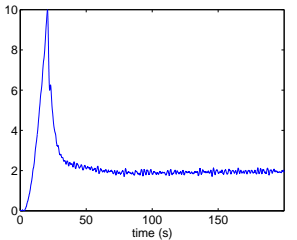

(d) Control input

Fig. 5. Rejection of temperature perturbation
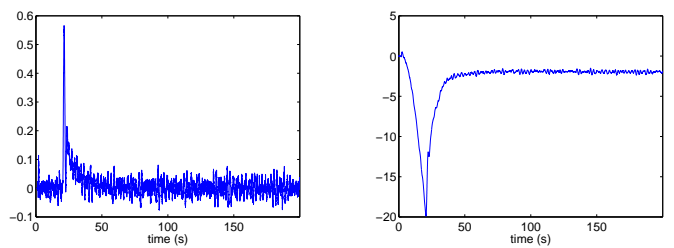

(a) Derivative estimation (b) Model part estimation $[\dot{y}]_{e}$ $[F]_{e}$

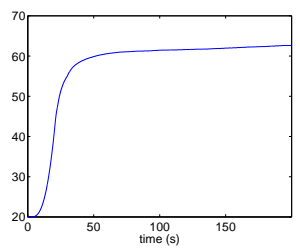

(c) Temperature $T$

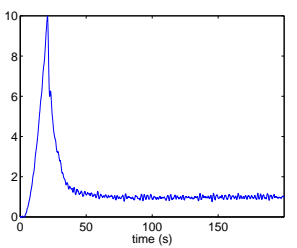

(d) Control input

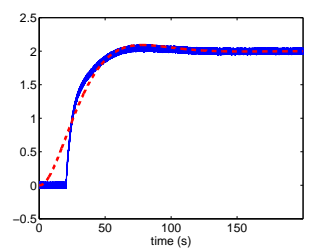

(e) Outputs (-), reference (-

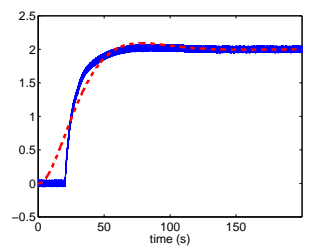

(e) Outputs (-), reference (-)

Fig. 6. Rejection of $\tau$ perturbation

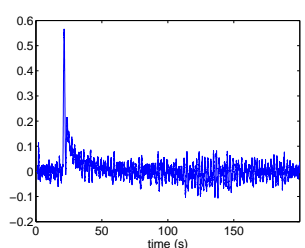

(a) Derivative estimation (b) Model part estimation $[\dot{y}]_{e}$

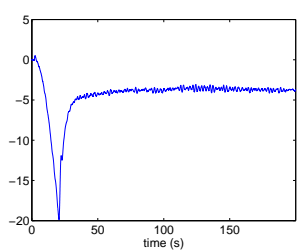

$[F]_{e}$

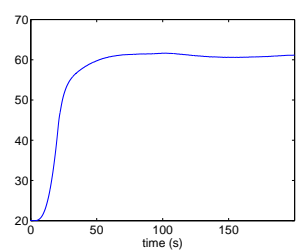

(c) Temperature $T$

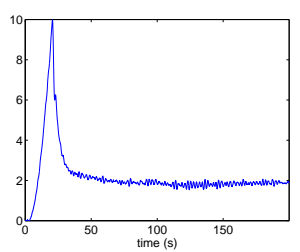

(d) Control input

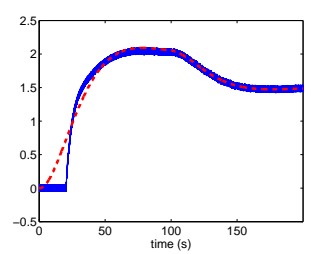

(e) Outputs (-), reference (-)

Fig. 7. Lower trajectory change
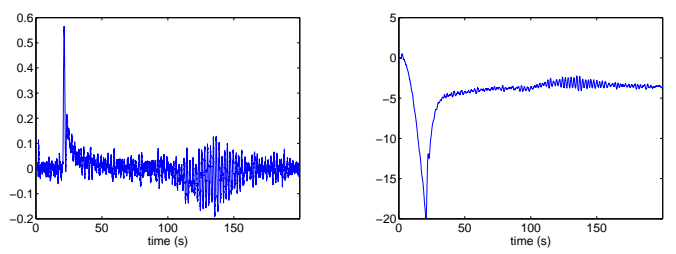

(a) Derivative estimation (b) Model part estimation $[\dot{y}]_{e}$ $[F]_{e}$

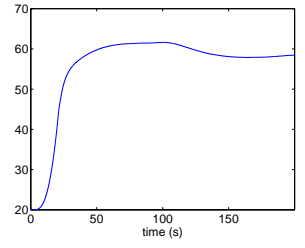

(c) Temperature $T$

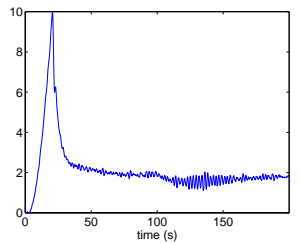

(d) Control input

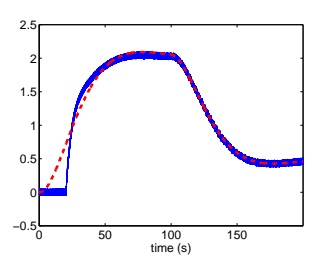

(e) Outputs (-), reference (-)

Fig. 8. Large trajectory change

We are very confident to be able to solve this problem and to show in a short time good experimental results using the model-free control in combination with on-line parameter estimation.

\section{REFERENCES}

S. Arbab Chirani, D. Aleong, C. Dumont, D. McDowell, and E. Patoor. Superelastic behavior modeling in shape memory alloys. Journal de Physique IV, 112:205-208, 


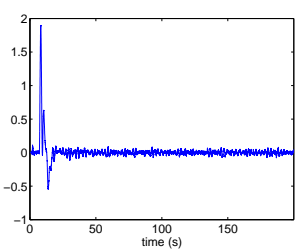

(a) Derivative estimation (b) Model part estimation $[\dot{y}]_{e}$

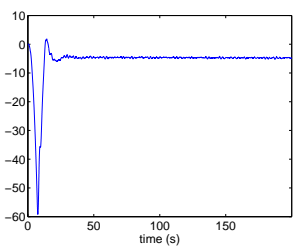

$[F]_{e}$

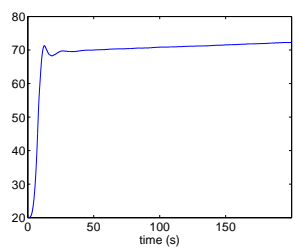

(c) Temperature $T$

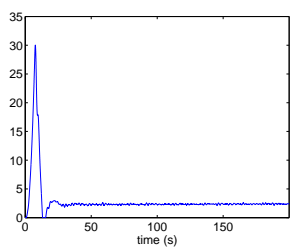

(d) Control input

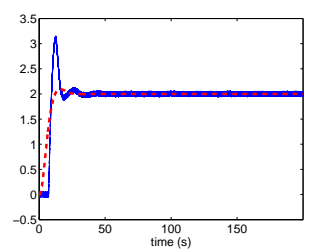

(e) Outputs (-), reference (-)

Fig. 9. Fast respond

2003.

H. Benzaoui, N. Chaillet, C. Lexcellent, and A. Bourjault. Nonlinear motion and force control of shape memory alloy actuators. Smart Structures and Materials 1999: Mathematics and Control in Smart Structures, 3667: 337-348, 1999.

C. Bouvet, S. Calloch, and C. Lexcellent. A phenomenological model for pseudoelasticity of shape memory alloys under multiaxial proportional and nonproportional loadings. European Journal of Mechanics A/Solids, 23: 37-61, 2004.

M. Calin, A. Bertsch, N. Chaillet, S. Zissi, S. Ballandras, J. C. Andre, A. Bourjault, and D. Hauden. Microrobots realized by microstereophotolithography and actuated byshape memory alloys. In Proceedings of the 1997 IEEE/RSJ Intelligent Robots and Systems, pages 33-34, Grenoble, France, September 1997.

E. P. Da Silva. Beam shape feedback control by means of a shape memory actuator. Materials and design, Elsevier Science Ltd, pages 1592-1596, 2007.

V. De Sars. Conception et commande d'une microstructure active à haute mobilité Application à la chirurgie mini invasive. Thèse de doctorat, Université Paris V, 2005.

S. M. Dutta, F. H. Ghorbel, and J. B. Dabney. Modeling and control of a shape memory alloy actuator. In International Symposium on Intelligent Control, pages 1007-1012, Limassol, Cyprus, 2005.

M. Fliess and H. Sira-Ramirez. An algebraic framework for linear identification. ESAIM : Control, Optimisation and Calculus of Variations, 9:151-168, 2003.

M. Fliess, J. Lévine, Ph. Martin, and P. Rouchon. Flatness and defect of non-linear systems: introductory theory and examples. Internat. J. Control, 61:1327-1361, 1995.

M. Fliess, R. Marquez, E. Delaleau, and H. Sira-Ramírez. Correcteurs proportionnels-intgraux gnraliss. ESAIM : Control, Optimisation and Calculus of Variations, 7:2341, 2002.

M. Fliess, C. Join, M. Mboup, and H. SiraRamìrez. Vers une commande multivariable sans modèle. In Proc. Conférence internationale francophone d'automatique (CIFA'06), $2006 \mathrm{a}$. (http://hal.inria.fr/inria-00001139/fr/).

M. Fliess, C. Join, and H. Sira-Ramirez. Vers une commande multivariable sans modèle. In Proc. 14th IFAC Symposium on System Identification (SYSID'06), 2006b. (http://hal.inria.fr/inria-00000824/fr/).

M. Fliess, C. Join, and H. Sira-Ramírez. Nonlinear estimation is easy. Int. J. Modelling, Identification and Control, 2007. To appear (http://hal.inria.fr/inria-00158855/fr/).
V. Hagenmeyer and E. Delaleau. Robustness analysis of exact feedforward linearization based on differential flatness. Automatica J. IFAC, 39:1941-1946, 2003a.

V. Hagenmeyer and E. Delaleau. Exact feedforward linearization based on differential flatness. Internat. $J$. Control, 76:537-556, 2003b.

P. Hippe. Windup in Control Its Effects and Their Prevention. Series: Advances in Industrial Control. Springer-Verlag, 2006.

C. Join, J. Masse, and M. Fliess. Commande sans modèle pour l'alimentation de moteurs : résultats préliminaires et comparaisons. In Proc. $2^{e}$ Journées Identification et Modélisation Expérimentale (JIME'06), 2006. (http://hal.inria.fr/inria-00096695/fr/).

M. Kohl, B. Krevet, and E. Just. Sma microgripper system. Sens. Actuators A Phys, 97-98:646-652, 2002.

S. Leclerc and C. Lexcellent. A general macroscopic description of the thermomechanical behaviour of shape memory alloy. I. Mech. Phys. Solids, Elsevier Science Ltd, 44(6):953-980, 1996.

D. R. Madill and D. Wang. Modeling and l2-stability of a shape memory alloy position control system. IEEE Trans. Control Systems Technology, 6(4):473-481, July 1998.

S. Majima, K. Kodama, and T. Hasegawa. odeling of shape memory alloy actuator and tracking control system with the model. IEEE Trans. Control Systems Technology, 9: 54-59, 2001.

M. Mboup, C. Join, and M. Fliess. A revised look at numerical differentiation with an application to nonlinear feedback control. In 15th Mediterrean Conference on Control and Automation (MED'07), 2007. (http://hal.inria.fr/inria-00142588/fr/).

E. Patoor, A. Eberhardt, and M. Berveiller. Micromechanical modelling of the shape memory behavior. Mechanics of phase transformation and shape memory alloys, 189 : 23-37, 1994.

J. Peirs, D. Reynaerts, and H. Van Brussel. A retrospective evolution of a sma microactuation. In In Proceedings of The International conference on new actuator, pages 77-80, 2002.

E. Shameli, A. Alasty, and H. Salaarieh. Stability analysis and nonlinear control of a miniature shape memory alloy actuator for precise applications. Mechatronics, 15:471486, 2005.

P. Sittner, D. Vokoun, G. N. Dayananda, and R. Stalmans. Recovery stress generation in shape memory ti50ni45cu 5 thin wires. Materials Science and Engineering, Elsevier Science Ltd, pages 298-311, 2000. 